\title{
CRISPR/Cas9 and cancer
}

\author{
N. Mimoune ${ }^{*}$, M. W. Bahouh ${ }^{\sharp}$ R. Baazizi, S. Boukhechem, D. Khelef \\ and R. Kaidi
}

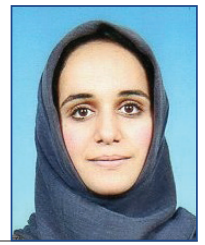

\section{Abstract}

CRISPR/Cas9 has become a powerful method for making changes to the genome of many organisms. First discovered in bacteria as part of an adaptive immune system, CRISPR/Cas9 and modified versions have found widespread use in genome engineering and in the activation or repression of gen expression. As such, CRISPR/Cas9 promises to accelerate cancer research by providing an efficient technology to dissect mechanisms of tumorigenesis, identify targets for drug development, and possibly arm cells for cellbased therapies. Here, we review the current applications of the CRISPR/Cas9 technology for cancer research and therapy. We highlight the impact of CRISPR/Cas9 in generating organoid and mouse models of cancer. Finally, we provide an overview of the first clinical trials applying CRISPR/Cas9 as a therapeutic approach against cancer.

Key words: CRISPR/Cas9; cancer; trial; application

\section{Introduction}

Cancer is one of the main causes of disease-associated mortality, with a rising incidence worldwide. Progress has been made in the prevention and treatment of many cancers, leading to prolonged survival or even cures. The main pillar of innovation in cancer therapy has been an improved understanding of the underlying tumor biology (Yin et al., 2019).

Recently, genome engineering was greatly accelerated by the development of CRISPR/Cas9 technologies (Mimoune et al., 2021). Since its first use in 2013 as a genome editing tool in mammalian cells, the CRISPR/Cas9 toolbox has been continuously expanded, enabling not only the modification of the genomic sequence of cells and organisms, but also the introduction of epigenetic and transcriptional modifications (Zhan et al., 2019).

In this review, we describe how CRISPR/Cas9 has opened new avenues for cancer research. In addition to its application as an effective screening method in functional cancer genomics, we outline how CRISPR/Cas9 can be used to explore the non-coding genome of cancer. Furthermore, we describe novel in vitro and in vivo cancer models that can be engineered by CRISPR/Cas9. Finally, we review the first clinical trials applying CRISPR as a therapy against cancer.

\footnotetext{
Nora MIMOUNE*, (Corresponding author, e-mail: nora.mimoune@gmail.com), National high school of veterinary medicine, Algiers, Bab-Ezzouar, Algeria, Institute of veterinary sciences, LBRA Laboratory, University of Blida 1, Algeria; Mohamed Wail BAHOUH, National high school of veterinary medicine, Algiers, Bab-Ezzouar, Algeria; Ratiba BAAZIZI, National High School of veterinary medicine, Bab Ezzouar, Algiers, Algeria; Said BOUKHECHEM, National Veterinary Institute, El-Khroub, Constantine, Algeria; Djamel KHELEF, National high school of veterinary medicine, Algiers, Bab-Ezzouar, Algeria; Rachid KAIDI, Institute of veterinary sciences, LBRA Laboratory, university of Blida 1, Algeria

\# These authors have contributed equally to this work
} 


\section{What is CRISPR?}

\section{Introduction to gene editing}

What is gene editing?

Gene editing involves inserting, deleting, modifying and replacing DNA in the genome of living organisms. It began in the 1970s, when scientists bombarded plants with radiation to cause random mutations. Gene editing started to show great promise with the incorporation of engineered gene-editing nucleases (Zink finger, Transcription Activator-Like Effectors, Meganucleases and CRISPR-Cas systems), which introduced the concept of targeted genome editing. The nucleases induce double-stranded breaks (DBS) into the targeted genomic locus. Nucleaseinduced DSBs can be repaired by one of two different pathways that operate in nearly all cell types and organisms: nonhomologous end-joining (NHEJ) and homology-directed repair (HDR). The NHEJ pathway involves the cell's own repair system by joining the DNA ends of a DSB, this process is prone to errors and can lead to the efficient introduction of insertion/deletion mutations (indels) of various lengths, which can disrupt gene function. The HDR pathway relies on a recombination of the DSBs ends and a homologous piece of DNA present in

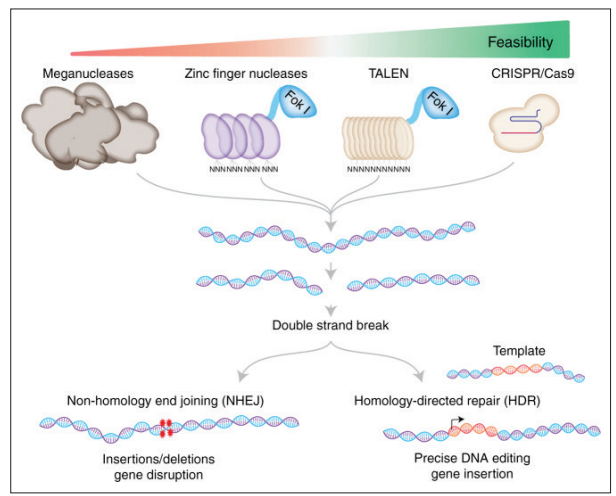

Figure 1. The basic working principle of major genome-editing technologies (Adli, 2018) the nucleus, this system can be used to introduce precise and desired mutations to the target genome (Figure 1) (Sander and Joung, 2014).

\section{Gene editing before CRISPR Zinc-Finger Nucleases}

Zinc-finger nucleases (ZFN) are fusions between the Cys2-His2 zincfinger protein and the cleavage domain of the Fok I restriction endonuclease that have been adopted as gene-targeting tools. In essence, they are targetable DNA cleavage reagents (Caroll, 2011; Gaj et al., 2016). Their discovery came when Chandrasekaran realized that the natural restriction enzyme, Fok I, has physically separable binding and cleavage activities, and that the cleavage domain could be guided and cutting could be redirected by replacing the recognition domains with alternative ones (Carroll, 2011). Of these alternatives, the Cys2His2 zinc fingers (ZFs) were the most useful, where usually 3 to 4 zinc fingers are fused to the Fok I cleavage domain, where each finger is comprised of 30 amino acids residues and interacts with three base pairs of DNA with occasional overlap from an adjacent domain. In order for the Fokl domain to cut DNA, it needs to dimerize (a chemical reaction that joins two molecular subunits, resulting in the formation of a single unit known as dimer. The dimer interface is weak, and to solve this problem and achieve better specificity, the best way to achieve cleavage is to construct two opposed sets of fingers directed to neighbouring sequences. When both sets are bound to their recognition sites, high local concentration facilitates the dimerization process and cleavage, resulting in a double-strand break (DSB) in the DNA (Caroll, 2011; Gaj et al., 2016). Despite great promise, the difficulty of constructing ZFs has hindered the widespread of this approach as it remains really challenging to create ZF-domains 
that can effectively recognize all DNA triplets outside specialized laboratories (Gaj et al., 2016).

\section{TALE Nucleases}

Transcription activator-like effectors (TALE) are bacterial proteins discovered in 2009, when scientists revealed the code behind TALE's capability to recognize DNA dps (DNA-binding proteins from starved cells), enabling the creation of custom TALE nucleases comprised of an amino-terminal TALE DNA-binding domain fused to a carboxy-terminal Fok I cleavage domain, enabling the editing of nearly any gene. In many ZFNs, the Fokl domain is responsible for dimerization and cutting, and unlike ZFs, which recognize DNA triplets, each TALE repeat recognizes only a single bp. Typically, TALEs are assembled to recognize from 12 to $20 \mathrm{dps}$ with more bases typically leading to higher genomeediting specificity. TALEs have a highly repetitive structure, making their delivery to the cell (through lentiviruses or a single adeno-associated virus) less efficient, though they have been reported to show improved specificity and reduced toxicity compared to some ZFNs. Engineering TALE arrays is much easier, reducing the amount of time and experience needed to assemble a functional nuclease (Boch et al., 2009; Gaj et al., 2016).

\section{Meganucleases}

The final members of the targeted nuclease family, also known as homing endonucleases, are highly specific DNA cleaving enzymes that have been engineered to be used for applications that require targeted gene modification (Stoddard, 2014). Discovered in the 1970s, these enzymes recognize and cleave long DNA sequences after making extensive sequence-specific contacts with their DNA substrate, showing superlative specificity. Their biggest limitation however is that the recognition and cleavage domains are not separable, and thus their repurposing and engineering is challenging, and their utility is limited (Stoddard, 2014; Gaj et al., 2016).

\section{CRISPR}

\section{What is CRISPR?}

CRISPR is a distinct array of 29-nucleotide repeat sequences separated by various 32-nt spacer sequences, discovered in 1987 when they were observed in certain bacteria. Since then, they have been found to be in $40 \%$ of all sequenced bacterial genomes and in nearly $90 \%$ of archaea. Although scientists have hypothesized about the function of the CRISPR sequences for years, the nature of their function has only recently been elucidated; in 2005, it was observed that the spacer sequences actually originate from phage genomes, and that these bacteria and archaea were immune to viruses with genomes identical to the sequences carried in the CRISPR arrays (Mojica et al., 2005; Bolotin et al., 2005; Makarova et al., 2011; Song et al., 2016).

Independently, several types of Cas genes were found adjacent to CRISPR sequences. When analysed in detail, the adjacent sequences were found to contain domains characteristic of several nucleases, a helicase, a polymerase and various RNA-binding proteins. These proteins were initially thought to constitute a novel DNA repair system in prokaryotic cells; however, with the observation that these Cas genes always neighboured the CRISPR array, and the spacer sequences were identical to fragments of the viral genome, a new hypothesis emerged: that the CRISPRCas system serves as a critical immune system to protect bacteria and archaea from pathogen invasions (Makarova et al., 2011; Song et al., 2016). This hypothesis was confirmed in 2007 when it was shown that that integration of a short phagespecific sequence into the CRISPR array of Streptococcus thermophilus conferred 
resistance to the cognate phage, and a complete loss of immunity towards the virus with the existence of a single mismatch between the CRISPR spacer and the target virus sequence (Barrangou et al., 2007).

\section{Classification and Associated proteins}

Given the fact that CRISPR is a fairly new technology, several of its properties and functions have only recently been understood or are yet to be elucidated. This is also the case for its associated proteins Cas, and as such, new classifications are released every few years. The current classification includes 2 classes, 6 types and 33 subtypes. Class one uses a complex of multiple Cas proteins to degrade foreign nucleic acids, includes Types I, III and IV, and is comprised of 16 subtypes. Class two uses a single large Cas protein to induce DNA degradation, includes Types II, V and VI, and 17 subtypes. The subtypes are characterized by a "signature gene" found almost exclusively in that category (Makarova et al., 2020).

\section{How does the CRISPR/Cas system work?}

The process in which the CRISPRCas systems mediate immunity against foreign genetic elements is divided into three stages - Adaptation, expression and interference - that fall under two subsystems. The first subsystem is highly conserved and is known as the "information processing" subsystem, and includes the adaptation stage, and the proteins involved in it (Cas1 and Cas2) are conserved between most prokaryotic beings. The second subsystem, called the "executive" subsystem, includes the expression and interference stages, and unlike the in first subsystem, the proteins in this subsystem vary greatly between different organisms (Makarova et al., 2011).

The adaptation stage revolves around the integration of short pieces of virus DNA sequences or plasmid into the CRISPR loci. This insertion process is known as "spacer acquisition" and it is triggered by viral invasions, where a single virus-derived resistanceconferring spacer, with a characteristic length of approximately $30 \mathrm{bp}$ at the leader side of a CRISPR locus. This is accompanied by the duplication of a repeat sequence, thus creating a new spacer-repeat unit (Barrangou et al., 2007; Makarova et al., 2011; McGinn and Marraffini, 2018).

The adaptation stage starts with the recognition of foreign nucleic acids or "protospacers". This step is of great importance for prokaryotic cells to avoid the integration of self-targeting spacers from the host's own chromosome into CRISPR, which leads to autoimmunity and even cell death. To avoid selftargeting, the CRISPR system employs various mechanisms that are biased to acquire only foreign genetic elements. CRISPR/Cas exploits the nature of the viral genome to stimulates spacer acquisition from double-strand breaks, carried out by relying on DNA repair machinery of the host, which binds only to the free ends of double stranded DNA to perform end resection during homologous recombination. This creates a biased recognition for viral DNA, as the bacterial chromosome is circular and lacks free DNA ends. Furthermore, the actions of the DNA repair system are inhibited by the presence of chi sites, which are eight nucleotide sequence motifs. The concentration of these Chi sites is much higher in host genome than in invader DNA, which constrains spacer acquisition from the host genome and differentiates self from non-self-nucleic acids. It remains unresolved if and how the DNA captured by the repair system can be used for spacer integration, with the leading hypothesis that Cas1 and Cas2 physically associates with the DNA repair component to directly uptake 
degradation products (Levy et al., 2015; McGinn and Marraffini, 2018).

Another important aspect of the recognition step is that the CRISPRCas system must select protospacers that can be functional spacers under type specific targeting requirements. This is to ensure the cleavage of foreign DNA and to prevent the cleavage of the spacer sequence in the CRISPR array during the interference stage. Different types have different requirements, i.e. in types I and II, the acquisition machinery preferentially samples genes flanked by a "protospacer-adjacent motif", though the two types achieve this differently: in type I, the Cas1-Cas2 complex has direct, sequence-specific interactions with the Protospacer Adjacent Motif (PAM) that biases acquisition to PAM-adjacent protospacers, while in type II, this selectivity for PAM-adjacent protospacers is the PAM-interacting domain of Cas9 that interacts directly with the Cas1-Cas2 complex (McGinn and Marraffini, 2018). In type III systems, the discrimination between self and non-self is achieved via the $5^{\prime}$ tag of the mature crRNA, which must not base pair with the target to enable degradation by the complex (Hille and Charpentier, 2016).

The expression stage involves the transcription process of the CRISPR locus to generate RNA-protein guides. All systems transcribe the CRISPR locus and generate the long primary transcript pre-crRNA which, depending on whether the CRISPR has repeats or not, may contain a series of secondary structures (hairpins). From here, the precrRNA is processed down and catalysed by endoribonucleases into short crRNAs, and the endoribonuclease either operate as a large complex like "Cascade" (CRISPR associated complex for antiviral defence) in the case of type I CRISPR-Cas system in Escherichia coli, or as a single enzyme, such as Cas6 in type III CRISPRCas system of the archaeon Pyrococcus furiosus.

In Type I systems, the complex remains associated with the Mature crRNA, while in Type III systems, Cas 6 passes the crRNA to a complex (a Type III complex)

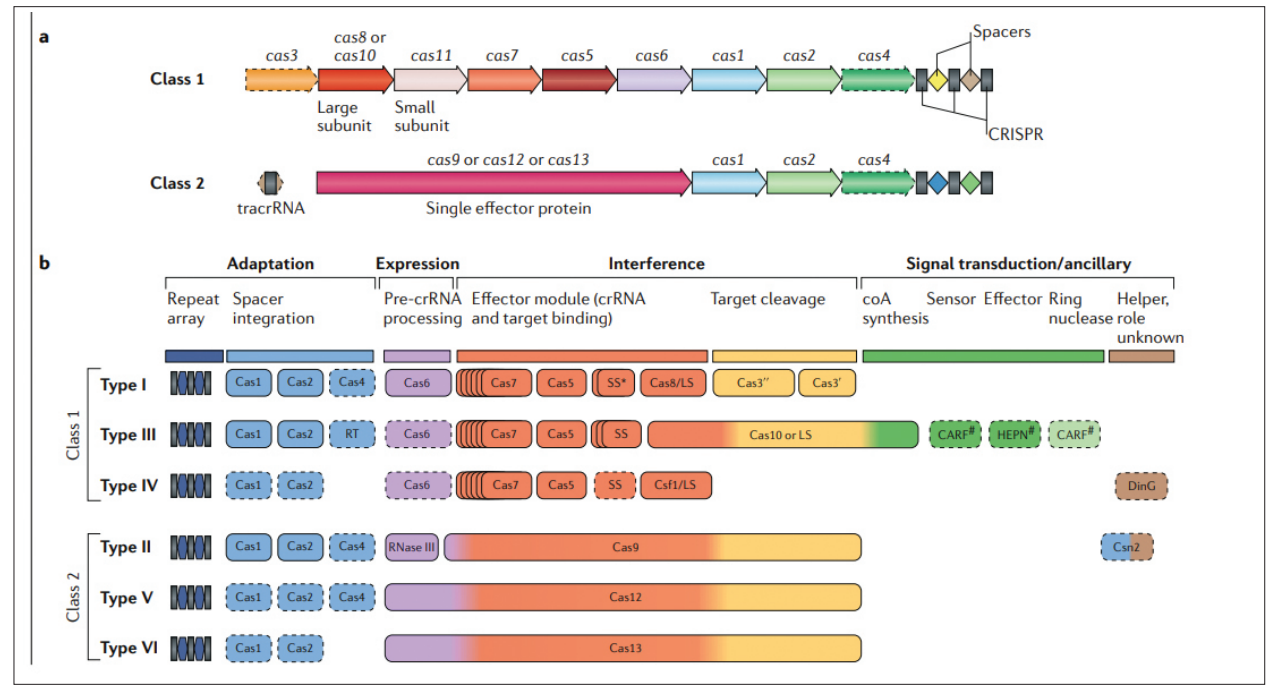

Figure 2. The two classes of CRISPR/Cas systems and their modular organization (Makarova et al. 2020) 
to undergo ruler-based sequenceunspecific processing and trimming at the $3^{\prime}$ end, yielding a mature crRNA with a defined $5^{\prime}$ end and variable $3^{\prime}$ end (Makarova et al., 2011; Rath et al., 2015). On the other end of the spectrum, there are Type II systems, which employ a very different approach for crRNA biogenesis. The maturation process is carried out by the host RNase III in the presence of a trans-encoded RNA (tracrRNA) that base pairs with the pre-crRNA. This process is stabilised by the Cas 9 protein and yields an intermediate form of crRNA, which is further processed by unknown mechanisms to reach the mature crRNA (Rath et al., 2015; Hille and Charpentier, 2016).

The interface stage starts with mature crRNAs, where in Type I CRISPR systems use Cascade-like complexes to bind the foreign DNA, then stimulate the Cas3 protein to carry out degradation, while Type II relies on a single protein (Cas9) to induce immunity. The Cas 9 protein, guided by the Trans-crRNA duplex, scans the cell and when it encounters the matching nucleic acid it performs a double strand break at a very specific site. Type III CRISPR systems are able to target both DNA and RNA, by relying on Cas10-Csm (types III-A and III-D) and Cas10-Cmr (types III-B and III-C) complexes, where the Cas10 cleaves the DNA while Csm3 and Cmr4 cleave the transcribed mRNA in type III-A and type III-B CRISPR-Cas systems, respectively (Figure 2) (Hille and Charpentier, 2016).

\section{How it is used in gene editing? Different CRISPR systems in gene editing}

The one common theme among all CRISPR systems is their ability to induce target specific DNA changes, whether through degradation or double stranded breaks. This qualify almost all of these systems for use in genome editing, though some systems are too complex for practical use. Nowadays, the Type II CRISPR-Cas9 system is the most widely used due to the simple "NGG" PAM sequence requirements (Figure 3) (Adli, 2018).

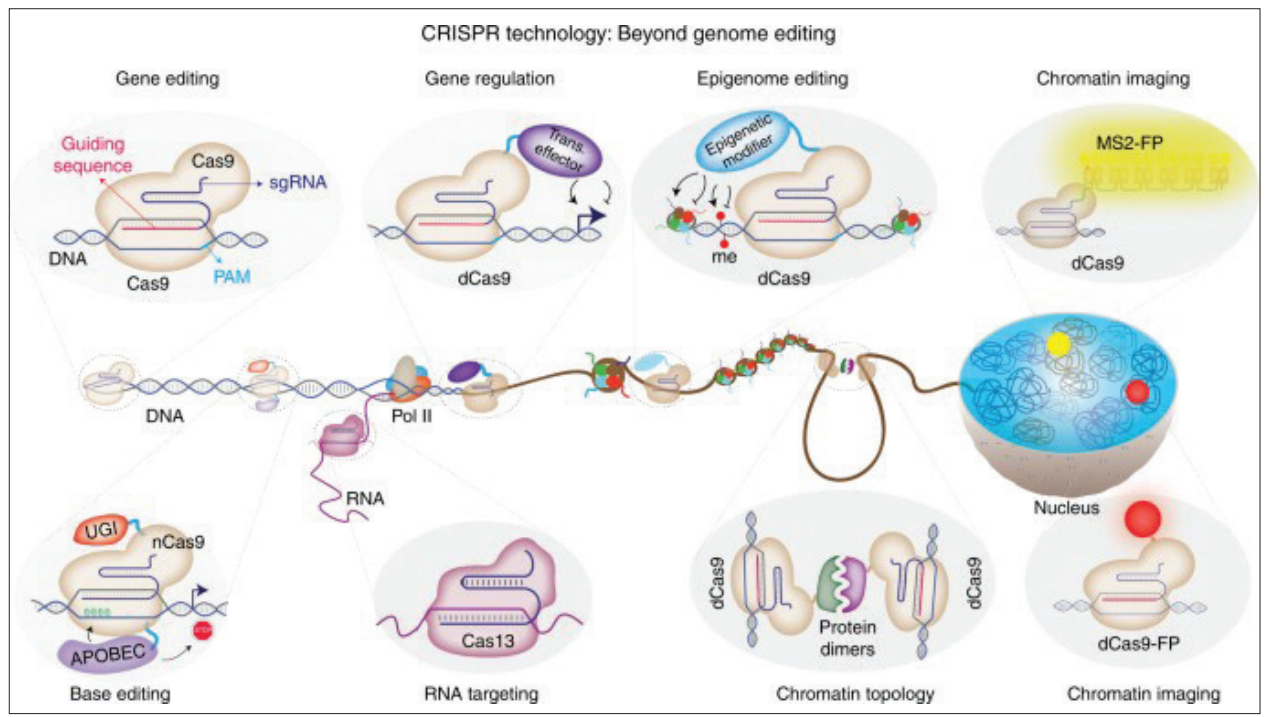

Figure 3. Major application areas of CRISPR/Cas-based technologies beyond genome editing (Adli, 2018) 


\section{The CRISPR/Cas9 system in gene editing}

The brilliance of the Type II CRISPR/

Cas9 system lies in in the mixture of versatility and simplicity it provides. This type relies on the Cas9 endonuclease of Streptococcus pyogenes (spCas9) to cleave the target DNA. The Cas9 protein is comprised of two cleaving domains, the $\mathrm{HNH}$ domain and the RuvC-like nuclease domain. The union of crRNA and tracrRNA "gRNA" guides the Cas9 to the target site to perform sequencespecific cleavage by simple interaction of crRNA by base pairing. The target sites must lie immediately $5^{\prime}$ of a PAM sequence that matches the canonical form 5'-NGG. This flexibility allows scientists to easily direct the Cas9 nuclease cleaving activity to any DNA sequence that is comprised of 18-24nt followed by NGG, simply by altering the first 18-24 nt of the gRNA (Sander and Joung, 2014; RodrigezRodrigez et al., 2019). Since 2012, when it was shown that the CRISPR/Cas9 system could be programable with chemical RNAs that retain the properties of the gRNA, the system has been implemented successfully for gene editing, and for the control of different types of biological systems, ranging from bacteria to in vitro human cancer cells and human pluripotent stem cells to whole organisms like the zebrafish, all while reducing the cost and time necessary for such alterations. Targeting of a new genomic locus for gene deletion, mutation and targeted insertion can be done rapidly through the generation of a gRNA, making CRISPR a powerful tool for research and drug development. However, being an acquired evolutionary arm that gives immunity mostly against viruses that frequently undergo mutations, a slightly less specific CRISPR system would be more advantageous to the host, and as such the CRISPR/Cas9 system allows cleavage at genomic locations that are partially complementary to the gRNA.
This hinders the accuracy of the system and remains one of the most important outstanding issues can result in undesired consequences (Sander and Joung, 2014; Adli, 2018; Rodrigez-Rodrigez et al., 2019).

Several approaches have been taken to increase the specificity of the CRISPR/ Cas9 system through the re-engineering of the existing spCas9. The first proof of concept came in a study that showed that a specific point mutation significantly increased the specificity of SpCas9. Another approach is the replacement of the traditional SpCas9 with a mutant variant (nSpCas9) which cuts a single strand through the inactivation of a nuclease domain RuvC or HNH. In this case, and much like the ZFN and TALEN methods, two nCas9 are needed to target opposite strands of DNA in close proximity, with each nCas9 guided by its own sgRNA reducing the off-target activity by 50 to 1,500 times. Furthermore, the fusion of dCas9 (catalytically inactive Cas9) with the DNA cleavage domain of Fok I, much like the previous approach with the presence of two distinct sgRNAs, substantially reduces off-target activity (Kleinstiver et al., 2016; Adli, 2018; Rodrigez-Rodrigez et al., 2019). Apart from re-engineering of the Cas9 protein, efforts also focused on modifying the sgRNA. Interestingly, it was proved that both increasing and decreasing the length of the sgRNA guiding sequence by a few base pairs enhanced targeting specificity (Cho et al., 2014; Fu et al., 2014).

\section{CRISPR/Cas9 beyond gene editing}

Due to its ease of use and flexibility, CRISPR/Cas9 provides a versatile tool for applications that are revolutionizing many genetic studies beyond gene editing. Soon after the discovery of dCas 9 and the ability to acquire catalytically inactive Cas9 that strongly binds to the DNA target sequence, interfering with the activity of other DNA binding 


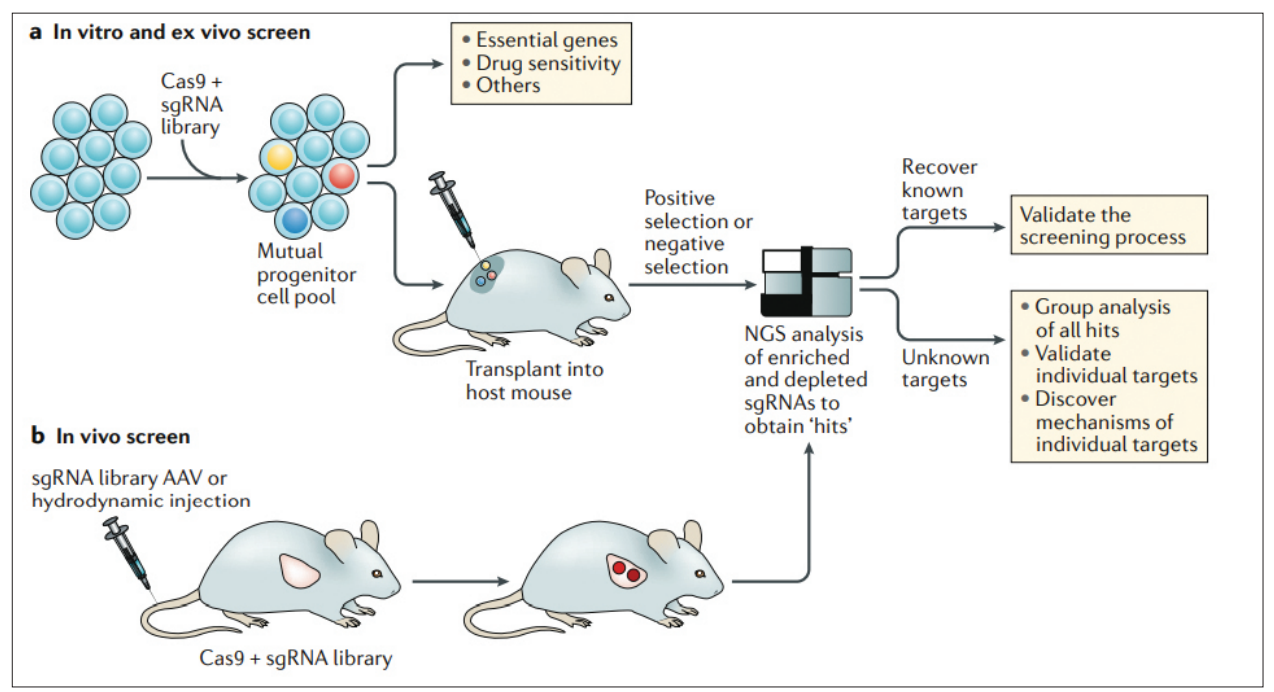

Figure 4. CRISPR for genetic screening (Yin et al., 2019)

proteins without initiating any cleavage processs, scientists have tried to exploit this property to regulate gene expression, mediated epigenome editing and screening (Figure 4).

Another use of dCas9 is in live cell chromatin imaging. The organization of chromatin structure in the 3D nuclear space plays a critical role in regulating lineage-specific gene expression, as such the dCas9 is used in fusion with fluorescent proteins or labels to target specific and repetitive regions enabling their imaging. Non-repetitive region imaging is more challenging due to interference of background fluorescence signals (free-floating fluorescently labelled dCas9 proteins). Typically, this requires transfection of as many as 26-36 unique sgRNAs to achieve live cell imaging of a non-repeat genomic region (Adli, 2018). Chromatin imaging allows for a better understanding of their 3D structure, but beyond imaging, one of the most exciting applications for the CRISPR/Cas9 system is chromatin topology. This involves engineering artificial chromatin loops between regulatory genomic regions, providing a means to manipulate endogenous chromatin structures to understand their function and contribution to gene expression. An elegant way to achieve this is through the fusion of two dimerisable protein domains ABI1 and PYL1 (taken from the plant-based abscisic acid signalling pathway) with two dCas9. This forces chromatin loop formation between the distal enhancer and promoter regions, allowing for targeted chromatin topology (Figure 5) (Morgan et al., 2017; Adli, 2018).

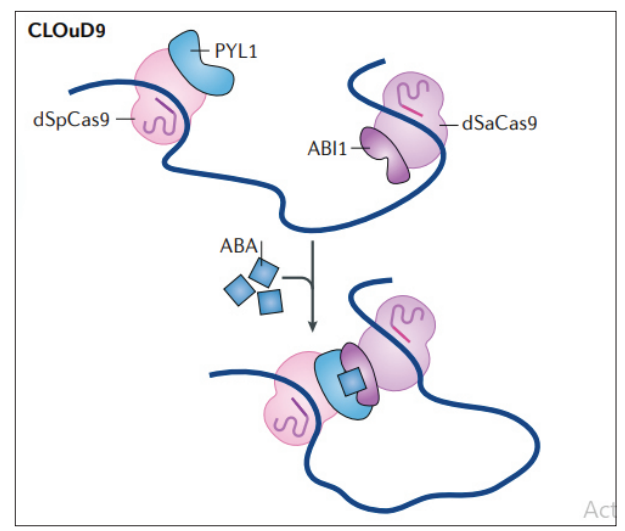

Figure 5. CRISPR use in chromatin topology (Pickar-Oliver and Gersbach, 2019) 


\section{CRISPR and Oncology}

Given its extreme versatility and ease of use, the CRISPR/Cas9 system represents an extraordinary therapeutic potential for treating different diseases originating from known genetic dysfunctions, and facilitating our understanding of the ambiguous aspects of complex genetic diseases through the creation of cell or animal models. Current preclinical research on genome editing primarily concentrates on viral infections, cardiovascular diseases (CVDs), metabolic disorders, primary defects of the immune system, haemophilia and muscular dystrophy. However, genetic diseases and their therapeutic trials cannot be discussed without addressing cancer (Yin et al., 2019; Li et al., 2020). Ever since it was discovered that cancer originate from DNA changes, scientists have been attempting to find ways to comprehend and correct these mutations. Although several technological advancements have been made regarding gene editing and regulation over the years, none have been satisfactory enough, up until the conception of CRISPR in 2013. The system has taken cancer research by storm, revolutionizing several fields of oncology from Cancer modelling and screening to immunotherapy and treatment (National Cancer Institute, 2020).

\section{CRISPR for cancer modelling}

To treat cancer, we first have to conceptualize it, by identifying the genes and their mutations that initiate carcinogenesis and drive tumour progression in genetically tractable models. This is of a great importance for the development of clinically effective therapeutics. Whole-genome sequencing of cancer cells reveals the multiple point drive mutations and as such, cellular and animal models, can be established using these data in order to understand the molecular mechanisms underlying tumorigenic responses. This was a lengthy and costly process that required laborious gene targeting, hindering the advancement of cancer research. However, his limitation is now alleviated by the CRISPR/Cas9 system, which in recent years have been extensively used to produce cancer models (Ratan et al., 2018; Yin et al., 2019).

\section{Cellular Modelling}

Because it relies on the gRNA rather than protein-DNA interactions, the CRISPR/Cas9 system allows for fast yet inexpensive modelling of mammalian cells with single genetic alteration. This has substantially accelerated pharmacological studies of targeted therapies, especially regarding the validation of gene function and their involvement in tumorigenesis (Yin et al., 2019). An important example is the MELK controversy. Maternal embryonic leucine zipper kinase (MELK) is an enzyme encoded by the MELK gene, and it was believed that the MELK enzyme is essential for cancer proliferation. However, through the CRISPR-mediated silencing of the MELK gene, it was found to have no effect on cancer cells growth and as such is fully dispensable (Huang et al., 2017; Lin et al., 2017; Yin et al., 2019). Another example involves the gene with the most mutations in human cancer: the p53 tumor suppressor gene. CRISPR/ Cas9 was used to validate the p53reactivating molecules nutlin and RITA, which were both initially identified as compounds that exhibit antiproliferative activity in tumour cells with an active p53, but not in p53-mutated or p53deleted cells, nutlin was confirmed to inhibit tumour proliferation via a p53dependent mechanism, but the activity of RITA was found to be p53-independent (Wanzel et al., 2016; Yin et al., 2019). Beyond the validation of certain genes identified in cancer research, the CRISPR/ Cas9 system could be used to disrupt functional genes or induce mutations 
to identify drug resistance properties in cultured cells. Furthermore, scientists use the CRISPR/Cas9 system to manipulate multiple genes in a cell to explore the genetic complexity of malignancies (Yin et al., 2019). But it doesn't stop there: the system's versatility allows for the study of tumour biology and its interaction with the micro-environment through genome edited organoids, which are tiny, self-organized three-dimensional tissue cultures derived from stem cells. Through the use of the CRISPR/Cas9 system, these organoids can be modelled to replicate much of the complexity of an organ, or to express selected aspects of it by producing only certain types of cells (Yin et al., 2019; Stemcell Technologies, 2020).

\section{In vivo modelling}

To fully understand how cancer operates, it is indispensable to study the interactions in whole organisms. The establishment of the CRISPR/Cas9 system in organism modelling has facilitated the study of cancers in living organisms, as the ability to edit the genome of somatic cells to induce driver mutations is more practical and cost efficient by margins than the manipulation of germline cells used before CRISPR. Moreover, the genetic manipulation of somatic cells resembles the naturally occurring driver mutations. The CRISPR/Cas9 system is introduced to the somatic cells through viral or plasmid delivery systems. The viruses used in the viral delivery system are: lentivirus or retrovirus. Lentiviral vectors enable stable expression of Cas9 and sgRNA and can therefore enable efficient gene editing in vivo. Lentiviral vectors have been widely used to deliver CRISPR locally in the target organs of interest to create animal models of brain, breast, colon, lung or pancreatic cancer. Despite their utility, lentiviruses have certain limitations, such as the fact that their delivery is surgery-dependent in some tissues like the brain, and the need to account for the random genomic integration of lentivirus to exclude lentivirus-induced off-target effects.

Adeno-associated virus and adenovirus: due to their broad- ranging tissue tropisms, AAS and adenoviruses have seen a great range of use in genome editing to generate animal models of cancer. These viruses can be delivered to the lungs quite easily with simple intranasal or intratracheal injection. However, the downside of their cargo capacity is that they are smaller than other viruses used for delivery, hindering their delivery range. Another limitation is their ability to generate a strong immune response in the liver, which might compromise the disease phenotype. Hydrodynamic injection or electroporation of plasmids: a high volume and pressure injection of the tail vein is a well-established method of delivering plasmids to the liver in rodents. This approach does not require any viruses for delivery, and and it has been shown to reliably model point mutations of tumour suppressor genes and oncogenes in the liver. However, this approach presents the lowest efficiency rate and the risk of liver damage (Yin et al., 2019).

\section{CRISPR and Immunotherapy}

For decades, surgery, chemotherapy, and radiotherapy have been the treatments of choice for cancer. However, in the last decade, the focus has been changing slowly but surely towards immunotherapy. CRISPR stands at the forefront of the technological advances that are facilitating the shift from general therapies to precise, targeted and immune induced therapies (Liu and Guo, 2018; Schirmacher, 2018; Yin et al., 2019; Esfahani et al., 2020; Waldman et al., 2020). CRISPR systems are being incorporated and adapted to improve the efficacy of immunotherapies by enhancing potency, reducing toxicity and manufacturing 
cost, and facilitating the discovery of new immunotherapeutic strategies (Yin et al., 2019). The CRISPR/Cas9 system has been used to establish the most promising approaches in cancer immunotherapy. For instance, in the Immune checkpoint blockade approach, scientists have relied on CRSIPR screening to identify checkpoint mediator genes and their functional expression. Such screening was used to point out the mechanisms by which the tumour cells avoid immune cells, enabling more effective engineering of the native T-Cell Receptor (TCRs) or Chimeric Antigen Receptors (CARs) (Yin et al., 2019; Khalaf et al., 2020).

\section{Direct Tumour targeting with the CRISPR/Cas9 system}

This approach could be described as going back to the basics of CRISPR/Cas9 gene editing capabilities. Cancer cells present specific genes which are usually the product of driver mutations that are absent in normal cells. This sparked the idea of using the CRISPR/Cas9 system to induce DSBs by relying on a pair of Cas9 nickases targeting two neighbouring cancer-specific genes. After that a suicidal gene (encoding a prodrug converting enzyme) with homology to the sequences surrounding the breakpoints is delivered via an adenoviral vector to enable its introduction into the genome via HDR (Chen et al., 2017; Yin et al., 2019).

\section{Conclusions}

Since its development as a genome editing tool, the CRISPR/Cas9 technology has revolutionized biology by providing a simple and versatile method to manipulate the genome and epigenome across a broad range of organisms. The potential of CRISPR/ Cas9 for both basic and translational cancer research is just beginning to unfold. In the future, pooled CRISPR screens will provide a comprehensive set of essential genes across most cancer cell lines. This resource, combined with the available information on the genetic and epigenetic characteristics of cancer cell lines, will enable the extensive identification of synthetic lethal interactions and facilitate the discovery of novel drug targets. The future use of CRISPR/Cas9 in translational medicine will largely depend on the ability to develop Cas9 variants with minimal or no off-target effect, and novel methods to improve the yet inefficient engineering of precise genetic changes by homology directed repair. Furthermore, future improvements of viral and non-viral delivery methods will be necessary to improve the in vivo application of CRISPR/Cas9, laying the ground for the therapeutic use of CRISPR in the future. In summary, the development of CRISPR/Cas9 technology has and will greatly accelerate cancer research in many areas.

\section{References}

1. ADLI, M. (2018): The CRISPR tool kit for genome editing and beyond. Nat. Commun. 9, 1911.

2. BARRANGOU, R., C. FREMAUX, H. DEVEAU, M. RICHARDS, P. BOYAVAL, S. MOINEAU, D. A. ROMERO and P. HORVATH (2007): CRISPR provides acquired resistance against viruses in prokaryotes. Science 315, 1709-1712.

3. BOCH, J., H. SCHOLZE, S. SCHORNACK, A. LANDGRAF, S. HAHN, S. KAY, T. LAHAYE, A. NICKSTADT and U. BONAS (2009): Breaking the Code of DNA Binding Specificity of TAL-Type III Effectors. Science 326, 1509-1512.

4. BOLOTIN, A., B. QUINQUIS, A. SOROKIN and S. D. EHRLICH (2005): Clustered regularly interspaced short palindrome repeats (CRISPRs) have spacers of extrachromosomal origin. Microbiology 151, 2551-2561.

5. CANCER RESEARCH INSTITUTE (2020, January): How Cellular Immunotherapies Are Changing the Outlook for Cancer Patients. Retrieved from Cancer research institute : https://www.cancerresearch.org/ immunotherapy/treatment-types/adoptive-celltherapy\#tcr

6. CARROLL, D. (2011): Genome engineering with zinc-finger nucleases. Genetics 188, 773-782.

7. CHEN, Z. H., Y. P. YU, Z.-H. ZUO, J. B. NELSON, G. K. MICHALOPOULOS, S. MONGA, S. LIU, G. TSENG and J.-H. LUO (2017): Targeting 
genomic rearrangements in tumor cells through Cas9-mediated insertion of a suicide gene. Nat. Biotechnol. 35, 543-550.

8. CHO, S. W., S. KIM, Y. KIM, J. KWEON, H. S. KIM, S. BAE and J. S. KIM (2014): Analysis of off-target effects of CRISPR/Cas-derived RNA-guided endonucleases and nickases. Genome Res. 24, 132-141.

9. ESFAHANI, K., L. ROUDAIA, N. BUHLAIGA, S.V. DEL RINCON, N. PAPNEJA and W. H. MILLER (2020): A review of cancer immunotherapy: from the past, to the present, to the future. Curr. Oncol. 27, S87-S97.

10. FU, Y., J. D. SANDER, D. REYON, V. M. CASCIO and J. K. JOUNG (2014): Improving CRISPR-Cas nuclease specificity using truncated guide RNAs. Nat. Biotechnol. 32, 279-284.

11. GAJ, T., S. J. SIRK, S. L. SHUI and J. LIU (2016): Genome-Editing Technologies: Principles and Applications. Cold Spring Harb. Perspect. Biol. 8(12), a023754.

12. HILLE, F. and E. CHARPENTIER (2016): CRISPR-Cas: biology, mechanisms and relevance. Philosophical transactions of the Royal Society of London. Series B, Biol. Sci. 371, 20150496.

13. HUANG, H.-T., H.-S. SEO, T. ZHANG, Y. WANG, B. JIANG, Q. LI and S. DHE-PAGANON (2017): MELK is not necessary for the proliferation of basallike breast cancer cells. eLife 6, e26693.

14. KHALAF, K., K. JANOWICZ, M. DYSZKIEWICZKONWIŃSKA, G. HUTCHINGS, C. DOMPE, L. MONCRIEFF, M. JANKOWSKI, M. MACHNIK, U. OLEKSIEWICZ, I., KOCHEROVA et al. (2020): CRISPR/Cas9 in Cancer Immunotherapy: Animal Models and Human Clinical Trials. Genes 11, 921.

15. KLEINSTIVER, B. P., V. PATTANAYAK, M. S. PREW, S. Q. TSAI, N. T. NGUYEN, Z. ZHENG, J. K. JOUNG (2016): High-fidelity CRISPR-Cas9 nucleases with no detectable genome-wide offtarget effects. Nature 529, 490-495.

16. LEVY, A., M. G. GOREN, I. YOSEF, O. AUSTER, M. MANOR, G. AMITAI, R. EDGAR, U. QIMRON and R. SOREK (2015): CRISPR adaptation biases explain preference for acquisition of foreign DNA. Nature 520, 505-510.

17. LI, H., Y. YANG, W. HONG, M. HUANG, M. WU and X. ZHAO (2020): Applications of genome editing technology in the targeted therapy of human diseases: mechanisms, advances and prospects. Signal Transduct. Target Ther. 5, 1.

18. LIN, A., C. J. GIULIANO, N. M. SAYLES and J. M. SHELTZER (2017): CRISPR/Cas9 mutagenesis invalidates a putative cancer dependency targeted in on-going clinical trials. eLife 6, e24179.

19. LIU, M. and F. GUO (2018): Recent updates on cancer immunotherapy. Precis. Clin. Med. 1, 65-74.

20. MAKAROVA, K. S., D. H. HAFT, R. BARRANGOU, S. J. BROUNS, E. CHARPENTIER, P. HORVATH, S. MOINEAU, F. J. M. MOJICA, U. I. WOLF, A. F. YAKUNIN, J. VAN DER OOST, E. V. KOONIN (2011): Evolution and classification of the CRISPRCas systems. Nat. Rev. Microbiol. 9, 467-477.
21. MAKAROVA, K. S., Y. I. WOLF, J. IRANZO, S. A. SHMAKOV, O. S. ALKHNBASHI, S. J. BROUNS and E. V. KOONIN (2020): Evolutionary classification of CRISPR-Cas systems: a burst of class 2 and derived variants. Nat. Rev. Microbiol. 18, 67-83.

22. MCGINN, J. and L. A. MARRAFFINI (2019): Molecular mechanisms of CRISPR-Cas spacer. Nat. Rev. Microbiol. 17, 7-12.

23. MIMOUNE, N., O. BENADJEL, R. BAAZIZI, D. KHELEF and R. KAIDI (2021): CRISPR/Cas9 uses: a review. Vet. stn. 52, 369-386.

24. MOJICA, F., C. DÍEZ-VILLASEÑOR, J. GARCÍAMARTÍNEZ and E. SORIA (2005): Intervening sequences of regularly spaced prokaryotic repeats derive from foreign genetic elements. J. Mol. Evol. 60, 174-182.

25. MORGAN, S. L., N. C. MARIANO, A. BERMUDEZ, N. L. ARRUDA, F. WU, Y. LUO, G. SHANKAR, L. JIA, H. CHEN, J.-F. HU, A. R. HOFFMAN, C. HUANG, S. J. PITTERI and K. C. WANG (2017): Manipulation of nuclear architecture through CRISPR-mediated chromosomal looping. Nat. Commun. 8, 15993.

26. NATIONAL CANCER INSTITUTE (2020, July 27): How CRISPR Is Changing Cancer Research and Treatment. Retrieved from National cancer institute: https://www.cancer.gov/news-events/ cancer-currents-blog/2020/crispr-cancer-researchtreatment

27. PICKAR-OLIVER, A. and C. A. GERSBACH (2019): The next generation of CRISPR-Cas technologies and applications. Nat. Rev. Mol. Cell Biol. 20, 490-507.

28. RATAN, Z. A., Y.-J. SON, M. F. HAIDERE, B. M. UDDIN, M. A.YUSUF, S. B. ZAMAN, J. H. KIM, L. A. BANU and J. Y. CHO (2018): CRISPR-Cas9: a promising genetic engineering approach in cancer research. Ther. Adv. Med. Oncol. 10, 1-15.

29. RATH, D., L. AMLINGER, A. RATH and M. LUNDGREN (2015): The CRISPR-Cas immune system: Biology, mechanisms and applications. Biochimie 117, 119-128.

30. RODRÍGUEZ-RODRÍGUEZ, D. R., R. RAMÍREZSOLÍS, M.A. GARZA-ELIZONDO, M. D. GARZARODRÍGUEZ and H. A.BARRERA-SALDAÑA (2019): Genome editing: A perspective on the application of CRISPR/Cas9 to study human diseases (Review). Int. J. Mol. Med. 43, 1559-1574.

31. SANDER, J. D. and J. K. JOUNG (2014): CRISPRCas systems for editing, regulating and targeting genomes. Nat. Biotechnol. 32, 347-355.

32. SONG, G., M. JIA, K. CHEN, X. KONG, B. KHATTAK, C. XIE, A. LI and L. MAO (2016): CRISPR/Cas9: A powerful tool for crop genome editing. The Crop Journal 4, 75-82.

33. STEMCELL TECHNOLOGIES (2020, July): CRISPR-Cas9 Genome Editing of Human Intestinal Organoids. Retrieved from STEMCELL: https:// www.stemcell.com/crispr-cas9-genome-editing-ofintestinal-organoids-using-arcitect-and-intesticult. html 
34. STODDARD, B. (2014): Homing endonucleases from mobile group I introns: discovery to genome engineering. Mobile DNA 5, 7.

35. WALDMAN, A. D., J. M. FRITZ and M. J. LENARDO (2020): A guide to cancer immunotherapy: from $\mathrm{T}$ cell basic science to clinical practice. Nat. Rev. Immunol. 20, 651-668.

36. WANZEL, M., J. B. VISCHEDYK, M. P. GITTLER, N. GREMKE, J. R. SEIZ, M. HEFTER, M. NOACK, R. SAVAI, M. MERNBERGER, J. P. CHARLES et al.
(2016): CRISPR-Cas9-based target validation for p53-reactivating model compounds. Nat. Chem. Biol. 12, 22-28.

37. YIN, H., W. XUE and D. G. ANDERSON (2019): CRISPR-Cas: a tool for cancer research and therapeutics. Nat. Rev. Clin. Oncol. 16, 281-295.

38. ZHAN, T., N. RINDTORFF, J. BETGE, M. P. EBERT and M. BOUTROS (2019): CRISPR/Cas9 for cancer research and therapy. Semin. Cancer Biol. 55, 106-119.

\section{CRISPR/Cas9 i rak}

Nora MIMOUNE, National high school of veterinary medicine, Algiers, Bab-Ezzouar, Algeria, Institute of veterinary sciences, LBRA Laboratory, University of Blida 1, Algeria; Mohamed Wail BAHOUH, National high school of veterinary medicine, Algiers, Bab-Ezzouar, Algeria; Ratiba BAAZIZI, National High School of veterinary medicine, Bab Ezzouar, Algiers, Algeria; Said BOUKHECHEM, National Veterinary Institute, El-Khroub, Constantine, Algeria; Djamel KHELEF, National high school of veterinary medicine, Algiers, Bab-Ezzouar, Algeria; Rachid KAIDI, Institute of veterinary sciences, LBRA Laboratory, university of Blida 1, Algeria

CRISPR/Cas9 postala je snažna metoda za unošenje promjena $u$ genom brojnih organizama. Prvi put otkriven u bakterijama kao dio prilagodljivog imunološkog sustava, CRISPR/Cas9 i njegove modificirane verzije $\mathrm{u}$ širokoj su uporabi u inženjeringu genoma i aktivaciji ili suzbijanju ekspresije gena. Kao takav, CRISPR/Cas9 obećava ubrzanje istraživanja raka, osiguravajući učinkovitu tehnologiju za analizu mehanizama tumorogeneze, identifikaciju ciljeva za razvoj lijekova i moguće "naoružanje“ stanica za terapije na bazi stanica. U ovom je radu dat pregled današnjih primjena CRISPR/Cas9 tehnologije $\mathrm{u}$ istraživanju i liječenju raka, a naglašen je utjecaj CRISPR/Cas9 u generiranju organoidnih modela raka i modela raka miša. Na kraju, dat je pregled prvih kliničkih ispitivanja koja primjenjuju CRISPR/Cas9 kao terapijski pristup protiv raka.

Ključne riječi: CRISPR/Cas9, rak, ispitivanje, primjena 